\title{
DESIGUALDAD SALARIAL EN ARGENTINA. UNA INTERPRETACIÓN CON BASE EN CALIFICACIONES OCUPACIONALES ${ }^{\circ}$
}

\author{
WAGE INEQUALITY IN ARGENTINA. AN INTERPRETATION \\ BASED ON OCCUPATIONAL SKILLS
}

María Celeste Gómez*

enviado: 20 septiembre 2019 - aceptado: 23 junio 2020

\section{Resumen}

En este artículo se examina el rol de las calificaciones laborales en la desigualdad salarial de Argentina, definiendo calificaciones no solo mediante la dimensión educativa, sino principalmente la ocupacional. Se estiman primas a las calificaciones vía regresión por cuantiles durante 2004, 2009 y 2014, incluyendo interacciones con género. Los resultados revelan caídas en las brechas entre premios por calificación y en los niveles de desigualdad hacia dentro de dichas categorías, combinadas con menores retornos a la educación en salarios más altos. Ambos efectos confirman la hipótesis de que las calificaciones laborales se vinculan directamente con la desigualdad salarial y que en el período se mitigan las desigualdades, con una mejor posición de trabajadores operativos, en relación a técnicos y profesionales.

Palabras clave: desigualdad, salarios, calificaciones, educación, Argentina. Código JEL: J24, I24, D63, C21.

Gómez, M. C. (2020). Desigualdad salarial en Argentina. Una interpretación con base en calificaciones ocupacionales. Estudios económicos, 37 (75), 27-49.

* Facultad de Ciencias Económicas. Centro de Investigaciones en Ciencias Económicas (UNC-CONICET). Becaria de la Secretaría de Ciencia y Tecnología (UNC). Correo electrónico: mcelestegomez. arg@gmail.com. Este artículo forma parte del proyecto de tesis doctoral de la autora 


\begin{abstract}
In this article we examine the role of workers' skills in Argentina's wage inequality, defining them not only through the educational dimension but mainly through the occupational aspect. Skills premiums are estimated through quantile regression during 2004, 2009 and 2014, including gender interactions. Our findings reveal falls in the gaps between skill premiums and in the levels of inequality within these categories, combined with lower returns to education in higher wages. Both effects confirm the hypothesis that labor skills are directly linked to wage inequality, and that inequalities are mitigated during the period, with a better position of operational workers in relation to technicians and professionals.
\end{abstract}

Keywords: inequality, wages, skills, education, Argentina.

JEL Codes: J24, I24, D63, C21. 


\section{INTRODUCCIÓN}

Desde inicios de los años 2000, en el mercado laboral argentino se han combinado distintos cambios que han repercutido en su composición, su naturaleza y sus reglas de determinación de salarios. Estas transformaciones se dieron a la par de la evolución de la macroeconomía en general, la que estuvo signada, al principio, por la recuperación en los niveles de actividad y, unos años después, con el estancamiento y la puja distributiva derivada de un contexto inflacionario. Entre 2004 y 2014, la tasa de desempleo se redujo en más de seis puntos porcentuales, de un $13.6 \%$ a un $7.3 \%$ y el salario creció casi un $40 \%$ en términos reales, con una caída notable en su dispersión. El coeficiente de Gini salarial pasó de 0.39 a 0.31 puntos y el ratio de salarios entre el décimo decil y el primero se contrajo de 6.6 en 2004 a 5.4 en 2014.

Los cambios registrados en el período profundizaron ciertas estructuras ocupacionales preexistentes y generaron modificaciones en su distribución salarial. Los asalariados con calificación operativa, que representaban alrededor del $57 \%$ del total de trabajadores en 2004, se incrementaron al $61 \%$ en 2014, y los trabajadores sin calificación mantuvieron su participación en alrededor del 18\%. Los técnicos pasaron de representar el $16 \%$ a cerca del $14 \%$ y los profesionales mantuvieron relativamente su participación en alrededor del 8\%. En términos distributivos, durante el período el salario medio de los profesionales representó 1.9 veces el promedio de todos los asalariados y el de los técnicos rondó 1.4 veces la media general. Entre los grupos con menores calificaciones, el salario de los operarios se ubicó en 0.83 veces el promedio y el de los trabajadores sin calificación en 0.62. Las caídas en los índices de desigualdad se reflejan también en estos ratios, siendo menores en 2014 las brechas salariales entre niveles de calificación que una década atrás. El nivel educativo de los asalariados fue mejorando también en dicho lapso, siguiendo la tendencia de buena parte de los países durante las últimas décadas. Los trabajadores con estudios secundarios representaron en 2004 un $23.5 \%$, y un $29.7 \%$ en 2014. Aquellos con estudios superiores representaban un $18.8 \%$ en 2004 , alcanzando el $22 \%$ una década después. A la inversa, quienes no completaron la educación primaria o no tuvieron instrucción disminuyeron del 5.6\% al 3.1\%.

Teniendo en cuenta este panorama, distintas perspectivas teóricas se han empleado para indagar sobre los factores que ayudan a explicar el nivel y distribución de los salarios. No obstante, las calificaciones que los trabajadores poseen para desarrollar sus ocupaciones y tareas, en tanto insumos esenciales del proceso productivo, no han sido incorporadas como dimensiones centrales en la literatura sobre salarios desde una perspectiva microeconómica. Esto motiva a indagar qué rol juegan los premios a estas calificaciones en la determinación del 
salario como en su distribución. La hipótesis subyacente indica que los premios a las calificaciones ocupacionales inducen a una mayor desigualdad salarial, impactando positivamente en los ingresos laborales, pero en mayor medida entre quienes perciben los mayores salarios y poseen mayores calificaciones. Adicionalmente, se prevé que estos efectos se vean moderados hacia fines del período en un contexto de reducción de los parámetros de desigualdad.

\section{REVISIÓN DE LA LITERATURA}

Existen numerosas hipótesis con las que —desde una visión microeconómica - se ha intentado explicar al nivel y la distribución de salarios. De todas las que se enumeran aquí, solo el enfoque de las ocupaciones y las tareas le otorgan relevancia al conocimiento que el trabajador ha adquirido de una u otra forma para ejercer las mismas. A continuación, se resume brevemente la literatura sobre salarios, y luego se define un marco analítico en base a la perspectiva de calificaciones ocupacionales que motiva este artículo.

El punto de partida de la literatura sobre salarios es la teoría del capital humano, desarrollada por Schultz (1961), Becker $(1962,1964)$ y Mincer (1974). Para esta teoría, la educación es el principal impulsor de los ingresos laborales, de la desigualdad salarial y de la distribución de la masa salarial, bajo la concepción de que el individuo invierte en capital humano a través de la educación y la experiencia laboral. En un mercado laboral homogéneo y competitivo, los trabajadores son pagados de acuerdo a su productividad marginal y esta se incrementa invirtiendo en educación y en experiencia (Becker \& Chiswick, 1966; Joll, McKenna, McNabb \& Shorey, 1983). Mincer (1974) toma estos postulados y los desarrolla matemáticamente en lo que hoy se conoce como la "ecuación minceriana" de ingresos. En su modelo establece la hipótesis de una relación positiva entre la educación formal y la experiencia con los ingresos laborales. En base a este planteo, las "tasas de retorno" a la educación se definen como el ingreso adicional que reporta al trabajador un año más de educación, pago que el empleador realiza por la mayor productividad derivada de esa inversión en capital humano.

Desde una posición crítica sobre la teoría de capital humano, se destacan otras perspectivas que abordan los tópicos salariales, como los estudios que vinculan la educación y la tecnología, entre los que se encuentran modelos de oferta y demanda (Katz \& Murphy, 1992; Card \& Lemieux, 2001; Goldin \& Katz, 2009; Galiani, Cruces, Acosta \& Gasparini, 2017); o sobre sesgos a las calificaciones (Acemoglu, 2002; Card \& DiNardo, 2002; Gasparini \& Cruces, 2009). También, 
desde lo educativo, los modelos de sobreeducación, que introducen desbalances entre oferta y demanda de trabajo (Hartog, 2000; Quinn \& Rubb, 2006; De Santis, Gáname \& Moncarz, 2018).

Otros enfoques han considerado la heterogeneidad en las condiciones salariales de los trabajadores, como los estudios sobre segmentación laboral e informalidad, de particular relevancia para el contexto regional (Alzúa, 2008; Paz, 2013; Beccaria \& Groisman, 2015; Groisman, 2015; Paz, 2015). Dentro del segmento formal, la literatura que aborda el rol de las instituciones laborales (Alejo \& Casanova, 2016; Marshall, 2019; Gómez, 2020). La heterogeneidad puede surgir también de procesos de segregación vertical u horizontal, como en la discriminación salarial por género (Esquivel, 2007; Berniell, Berniell, de la Mata, Edo \& Marchionni, 2018; Marchionni, Gasparini \& Edo, 2019; Pal, 2019; Villanueva \& Lin, 2019).

De todas las hipótesis planteadas en la literatura para explicar la dispersión salarial, los enfoques sobre calificaciones ocupacionales han alcanzado un relativo menor desarrollo, a pesar de la importancia de las ocupaciones y las tareas como expresión del insumo laboral en el proceso productivo ${ }^{1}$. Desde la perspectiva ocupacional se critica la concepción neoclásica de mercados laborales homogéneos, donde la única fuente de diferencias viene dada por la escolarización del individuo. El argumento detrás de ello es que la educación ignora cualquier noción de especialización en los saberes adquiridos, así como la forma en la cual estos son apropiados por cada trabajador.

La literatura sobre ocupaciones parte de Roy (1951), quien desarrolla un modelo teórico sobre distribución ocupacional, con consecuencias en la distribución de ingresos en un mundo donde los individuos difieren en sus dotaciones de "habilidades específicas" para esas ocupaciones (Heckman \& Honore, 1990). Para el autor, la distribución del ingreso (y del producto) no es un mero accidente histórico o un resultado arbitrario, sino que depende críticamente de la distribución de los trabajadores entre las distintas ocupaciones disponibles en la comunidad ${ }^{2}$. En

1 Dos razones pueden explicarlo. Un escaso desarrollo del rol de las ocupaciones en el campo económico en general y un avance teórico relativamente reciente, el "enfoque de las tareas", popularizado en la última década.

2 El enfoque de las tareas surge de esta perspectiva, donde las ocupaciones se definen como grupos de tareas similares que requieren conjuntos de habilidades similares (Autor, Levy \& Murnane, 2003; Acemoglu \& Autor, 2011). Cada ocupación generará un producto empleando un vector particular de tareas, donde el valor productivo de cada tarea debe diferir según las ocupaciones (Autor \& Handel, 2013). 
línea con lo anterior, Polachek (1981) critica la teoría de capital humano que asocia calificaciones solo a su dimensión educativa y se refiere a montos de capital y no a los "tipos" de capital humano. El autor sostiene que incorporar estas diferencias permitirá una mejor explicación de la distribución de ingresos. Por otro lado, tanto Avent-Holt et al. (2019) como Kunst, Freeman y Oostendorp (2020) coinciden en que las ocupaciones son determinantes clave en la varianza salarial y las conectan necesariamente con las tareas laborales.

En virtud de lo anterior, surge la necesidad de incorporar al análisis de las calificaciones la dimensión ocupacional. El enfoque adoptado es una adaptación al de De Beyer y Knight (1989). Los autores consideran que tanto las características del individuo como las del puesto de trabajo son relevantes en la determinación de salarios, y las incluyen en la ecuación salarial combinando con la educación del individuo. La incorporación de estos atributos - cuyo sujeto es el trabajador, pero cuyo objeto es el puesto de trabajo - permite captar la naturaleza del individuo que desarrolla una actividad, posee un cuerpo de herramientas o habilidades para la realización de sus tareas y percibe una remuneración por ello. La definición oficial a la que suscribe esta investigación indica que:

... la calificación ocupacional se trata de una característica objetiva del proceso productivo que determina los conocimientos y habilidades requeridos a las personas que lo ejercen y que, por ende, "califica" el grado de complejidad de las ocupaciones y no de las personas. El hecho de que las ocupaciones sean ejercidas por personas y que por ello generalmente se hable de mayor o menor "calificación" en relación a estas es una consecuencia de la complejidad de sus tareas y no de su formación educativa o su experiencia laboral (INDEC, 2018).

El premio a un nivel de calificación determinada puede estimarse como el salario adicional que marginalmente obtiene el trabajador por poseer las habilidades y/o capacidades necesarias para el desarrollo de las tareas asociadas a un puesto de trabajo. La incorporación de las categorías de calificación —operativa, técnica o profesional - como variables de peso en la ecuación salarial es un avance analítico para los estudios sobre desigualdad salarial, los cuales se han concentrado —en la mayoría de los casos- en los niveles educativos ${ }^{3}$.

3 En INDEC (2018) se definen las ocupaciones. Operativas: implican tareas secuenciales y variadas que suponen atención, rapidez y habilidades manipulativas y conocimientos específicos sobre propiedades de los objetos e instrumentos utilizados. Técnicas: involucran tareas múltiples, diversas y de secuencia cambiante, suponen habilidades manipulativas y conocimientos teóricos específicos sobre 
La literatura sobre Argentina ha seguido, en general, el enfoque neoclásico que resalta los retornos a la educación como fuente de dispersión salarial, aunque con matices que hacen alusión a las ocupaciones y los puestos de trabajo. Aliaga y Montoya (1998) introducen el concepto de empleabilidad, por el que no solo el trabajador decide participar del mercado laboral, sino que también es "seleccionado" entre otros para ocupar un puesto laboral. La conexión entre oferta y demanda laboral se expresa - entre otros modos - en el hecho de que empleador paga mayores salarios a aquellos trabajadores más calificados.

Algunos trabajos sobre Argentina combinan el análisis de los retornos a la educación con los premios a las calificaciones ocupacionales. Groisman y Marshall (2015) examinan ambos premios salariales, al igual que las brechas salariales interocupacionales, resaltando la importancia de interpretar no solo la configuración de la oferta laboral, sino también las motivaciones de la demanda ${ }^{4}$. Asimismo, Paz (2013) y Casal, Paz Terán y Paz (2016) analizan como determinantes los años de educación y la calificación laboral, entre otros. Otros autores incluyen las dimensiones ocupacionales en las ecuaciones salariales, si bien con foco en los retornos a la educación (Groisman, 2014; Beccaria \& Groisman, 2015; Paz, 2015; Alejo \& Casanova, 2016; Ciaschi, Galeano \& Gasparini, 2019). Estos estudios revelan una caída en los premios salariales en Argentina a lo largo de los años 2000 y comienzos de la década siguiente. Más allá de estos antecedentes, no se han encontrado desarrollos empíricos que profundicen el análisis en Argentina, lo cual abre el campo de estudio respecto a la naturaleza de las calificaciones y vínculo con la desigualdad salarial.

\section{DATOS Y METODOLOGÍA}

\section{II.1. Datos}

En este artículo se usan microdatos provenientes de la Encuesta Permanente de Hogares en su modalidad continua trimestral (EPHC), para los años 2004, 2009 y 2014. La EPHC representa cerca del 62\% de la población total de la Argentina y

las propiedades y características de los objetos e instrumentos de trabajo y de las reglas de los procesos involucrados. Profesionales: en ellas se realizan tareas múltiples, diversas y de secuencia cambiante, que suponen conocimientos teóricos de orden general y específico acerca de las propiedades y características de los objetos e instrumentos de trabajo y de las leyes y reglas que rigen los procesos.

4 Según los autores, pueden verificarse puestos que combinan niveles similares de calificación ocupacional con diferentes dotaciones educativas. 
sigue un esquema de panel rotativo, donde cada hogar es encuestado en un lapso total de seis trimestres. Para lograr mayor comparabilidad entre los años que involucra este estudio, fue necesario un trabajo de armonización previo sobre las bases de datos: a) los últimos aglomerados incorporados a los relevamientos desde el año 2006 no se incluyeron para así trabajar con 28 centros urbanos durante todo el período de análisis; b) se trabajó con asalariados del sector privado y público de tiempo completo (35 o más horas laborales semanales) de 18 a 64 años.

\section{II.2. Metodología}

En cuanto a la estrategia empírica, la versión de Mincer (1974) de las ecuaciones de ingreso muestran el valor que el mercado paga (premia) por las características observables del individuo. Además de la educación y la experiencia, se incluyen otros determinantes como el entrenamiento laboral, las calificaciones, el género, la región geográfica, la formalidad del empleo, etc. La especificación general de la ecuación de Mincer sigue la siguiente forma:

$$
w_{i}=\vartheta\left(k_{i}, e_{i}, h_{i}\right)+u_{i}
$$

Donde $w_{\mathrm{i}}$ indica el salario real (en logaritmos) para el individuo $\mathrm{i} ; \mathrm{k}_{\mathrm{i}}$ es el stock de capital humano medido en años de educación, $e_{i}$ es la experiencia laboral potencial (que se aproximará con la variable edad); $h_{i}$ otros factores observados del individuo; $\mathrm{y}_{\mathrm{i}}$ es el error aleatorio que captura características no observadas del individuo y se distribuye $\mathrm{N}\left(0, \sigma^{2}\right)$.

La variable dependiente en esta especificación es el salario horario de la ocupación principal y las covariables son los años de educación, tres variables dummy que indican la calificación ocupacional, la edad y su cuadrado, el género, y la formalidad en el empleo; d) se trabajó con ingresos a precios constantes con paridad de poder de compra del consumidor entre las regiones de Argentina (INDEC, 2002) ${ }^{5}$. Se incluyeron, además, variables de control por ramas de actividad, espacial (regiones geográficas de Argentina) y temporal (trimestres).

Con el objeto de indagar sobre los aspectos distributivos, la metodología empleada sigue la técnica de regresiones por cuantiles condicionales, método

5 Se utilizó el IPC Gran Buenos Aires publicado por INDEC para deflactar los ingresos en 2004, empalmado al IPC de la provincia de Salta en 2009 y 2014, el cual mostró altas correlaciones con otros índices provinciales, en períodos pre- y post-intervención al INDEC. 
usualmente usado en contextos distributivos. En este modelo, los cuantiles son modelados en función de la distribución de las características observables de los individuos. A diferencia de mínimos cuadrados ordinarios (estimación de la media condicional), aquí la regresión opera sobre diferentes puntos definidos de la distribución (Koenker \& Bassett, 1978). Siguiendo a Machado \& Mata (2005), para cada $\tau \in(0,1)$, el $\tau$-ésimo cuantil de la distribución salarial dado el vector de covariables, puede modelarse en:

$$
Q_{t}(w \mid x)=x^{\prime} b(\tau)
$$

Siendo b el vector de coeficientes de la regresión cuantílica. Además, $w$ indica el salario horario en logaritmos y $x$ el conjunto de atributos (incluyendo educación, edad, edad ${ }^{2}$ y calificaciones ocupacionales). La estimación de $b(\tau)$ se plantea como un problema de minimización:

$$
n^{-1} \sum_{i=1}^{n} \rho_{\tau}\left(w_{i}-x_{i}^{\prime} b\right) \quad \rho_{\tau}(u)=\left\{\begin{array}{c}
\tau u \text { en } u \geq 0 \\
(\tau-1) u \text { en } u<0
\end{array}\right\}
$$

En este caso se estimaron las correlaciones sobre una serie de percentiles representativos del salario (p10, p25, p50 o mediana, p75, y p90) para los años 2004, 2009 y 2014. También, en una segunda instancia se introdujo la variable género en interacción con las variables de calificación (educación y los tres niveles de calificación ocupacional), con la idea de identificar si la condición de género de los trabajadores está asociada a una diferente estructura de premios salariales. Para identificar si las diferencias entre los premios a distintos cuantiles resultan significativas, se realizaron regresiones intercuantílicas entre los cuantiles estimados. Respecto a las brechas entre calificaciones, se efectuaron test de Wald sobre los coeficientes en cada cuantil estimado. Por último, los errores estándar para todas las estimaciones se calcularon, a su vez, mediante la técnica de Bootstrap con 200 réplicas. En la siguiente sección se discuten los resultados.

\section{RESULTADOS EMPÍRICOS}

\section{III.1. Análisis Descriptivo}

Para contextualizar las dimensiones laborales incorporadas al análisis, la tabla 1 muestra los estadísticos descriptivos para las principales variables empleadas en las estimaciones. La tabla indica que el peso de los trabajadores con calificación operativa es fuertemente mayoritario, al reunir el 59\% de la muestra. En orden decreciente le siguen los trabajadores que no declaran calificación ocupacional 
alguna con el $17 \%$, aquellos con calificación técnica (15\%), y finalmente los profesionales $(8 \%)$. A su vez, el promedio de años de educación completos se aproxima a los doce, coincidiendo con el plazo mínimo estipulado de finalización de la escuela secundaria en Argentina.

Tabla 1. Descripción de las variables y estadísticos descriptivos

\begin{tabular}{|c|c|c|c|c|c|}
\hline Variable & Definición & Media & $\begin{array}{l}\text { Desv. } \\
\text { Est. }\end{array}$ & Mín & Max \\
\hline salario & $\begin{array}{l}\text { Salario horario de la ocupación } \\
\text { principal (en logaritmos). }\end{array}$ & 1,45 & 0,59 & $-1,27$ & 5,50 \\
\hline sincalif & $\begin{array}{l}(1 / 0) \text { si el/la trabajador/a no tiene } \\
\text { calificación ocupacional alguna }\end{array}$ & 0,17 & 0,38 & 0,00 & 1,00 \\
\hline oper & $\begin{array}{l}(1 / 0) \text { si el/la trabajador/a tiene } \\
\text { calificación operativa }\end{array}$ & 0,59 & 0,49 & 0,00 & 1,00 \\
\hline tecn & $\begin{array}{l}(1 / 0) \text { si el/la trabajador/a tiene } \\
\text { calificación técnica }\end{array}$ & 0,15 & 0,35 & 0,00 & 1,00 \\
\hline prof & $\begin{array}{l}(1 / 0) \text { si el/la trabajador/a tiene } \\
\text { calificación profesional }\end{array}$ & 0,08 & 0,27 & 0,00 & 1,00 \\
\hline educa & años de educación formal & 11,53 & 3,59 & 0,00 & 17,00 \\
\hline edad & edad del/a trabajador/a & 38,15 & 11,43 & 18,00 & 64,00 \\
\hline hombre & $(1 / 0)$ si es hombre & 0,70 & 0,46 & 0,00 & 1,00 \\
\hline formal & $(1 / 0)$ si está registrado & 0,83 & 0,38 & 0,00 & 1,00 \\
\hline
\end{tabular}

Fuente: elaboración propia con datos de la Encuesta Permanente de Hogares Continua (EPHC-INDEC).

Asimismo, la figura 1 muestra la distribución de trabajadores según los años dedicados a la educación formal. En ella, los años de educación muestran una distribución con múltiples modos, ubicando los tres principales, en orden de frecuencia, a los doce, a los siete y a los dieciocho años. Estos períodos coinciden con la finalización de los tres niveles principales definidos en el esquema educativo actual (nivel primario, secundario y universitario) ${ }^{6}$.

6 Para la construcción de la variable años de educación se tuvo en cuenta el impacto de las reformas del sistema educativo argentino. Para Alzúa, Gasparini y Haimovich (2015), la Ley Federal de Educación tuvo impacto moderado sobre empleo e ingresos laborales, y nulo si el análisis se centra en los jóvenes en condición de pobreza. 
Figura 1. Asalariados según años de educación. Argentina

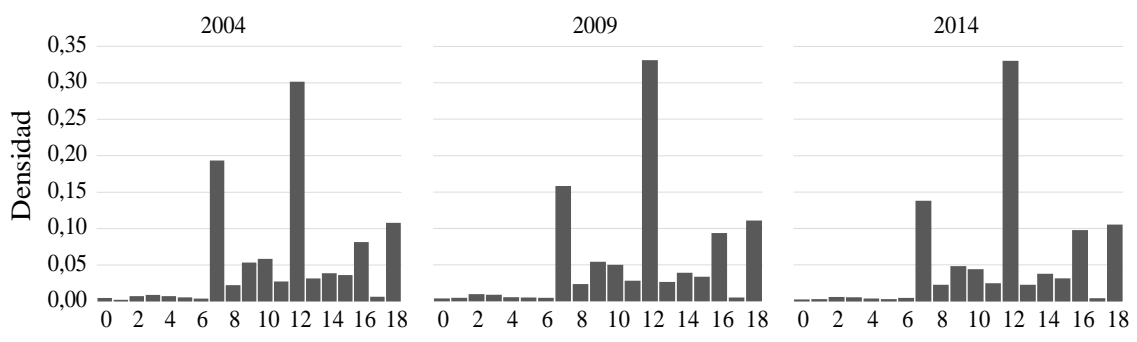

Fuente: elaboración propia con datos de la EPHC (INDEC).

Nota: hasta 18 años de educación.

En cuanto a la calificación para la tarea, la figura 2 muestra cómo los trabajadores con calificación operativa fueron aumentando entre 2004 y 2014, de un $57 \%$ a un $61 \%$ del total, mientras el resto de las categorías se mantuvieron relativamente estables o se redujeron. A lo largo del período, la desigualdad entre los trabajadores para igual nivel de calificación resulta mayor entre los asalariados profesionales que entre las personas sin calificación alguna (con un Gini de 0.31 y 0.29 , respectivamente). Esta distribución no es uniforme entre ambos géneros, si bien en ambos casos los trabajadores con calificación operativa son mayoría. La distribución de calificaciones entre mujeres resulta más armónica, ya que las operarias representan el $43 \%$ de la fuerza laboral femenina, mientras los operarios suman el $64 \%$ de los hombres. La participación de sectores como la industria o la construcción - con fuerte presencia masculina e incidencia en puestos operativosparecen incidir en estos resultados.

Figura 2. Asalariados según calificación ocupacional. Argentina

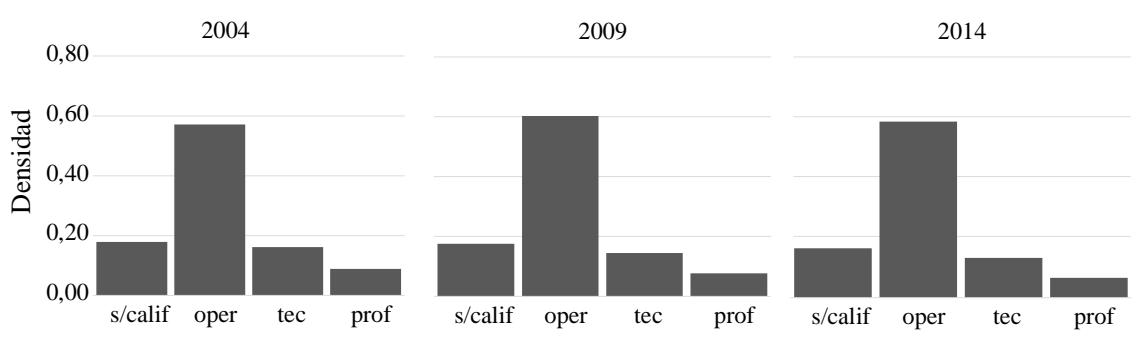

Fuente: elaboración propia con datos de la EPHC (INDEC).

Nota: $\mathrm{s} / \mathrm{calif}=$ sin calificación; oper=operario; técn=técnico; prof=profesional. 
Adicionalmente, se estimaron coeficientes de correlación de Spearman en las variables que definen calificación y el salario. Los resultados se muestran en la tabla 2.

Tabla 2. Correlación por rangos de Spearman. Argentina

\begin{tabular}{llll}
\hline & calificación ocupacional (niveles) & años de educación & edad \\
\hline Spearman (salario) & $0.39 * * *$ & $0.37 * * *$ & $0.23 * * *$ \\
\hline
\end{tabular}

Fuente: elaboración propia con datos de la EPHC (INDEC).

Las correlaciones presentadas tienen los signos y magnitudes esperadas y resultan significativas en todos los casos al 1\%. Las calificaciones ocupacionales, los años de educación y la edad están asociados positivamente con el salario horario, indicando que aumentos sucesivos en estas dimensiones están vinculados con mayores niveles salariales.

\section{III.2. Premios a las calificaciones ocupacionales}

En este apartado se presentan los resultados de la estimación de regresión por cuantiles condicionales para los años 2004, 2009 y 2014. En este modelo los coeficientes respectivos se aplican a lo largo de la muestra de trabajadores, sin distinguir por género.

En la tabla 3, a continuación, se muestran las primas salariales estimadas en base a las ecuaciones salariales, sobre los distintos puntos de la distribución salarial ${ }^{7}$. Los retornos a la educación y los premios a las calificaciones muestran los signos esperados en todos los cuantiles estimados y en los tres años analizados. Un año adicional de educación se corresponde con un mayor salario, independientemente de la posición del trabajador en la distribución salarial. Por otro lado, los premios a las distintas calificaciones ocupacionales siguen el orden esperado: a mayor nivel de calificación, mayor es el premio reportado, más allá del tramo de la distribución salarial que estemos considerando. En todos los casos se visualizan coeficientes positivos con un grado de significatividad del 1\%. El resto de las covariables muestran los resultados esperados. La edad, y su cuadrado, son

$7 \quad$ Las correlaciones de las variables discretas se calculan mediante la regla $\left(e^{b i}-1\right) * 100 \%$, denotando en bi los coeficientes en tablas. Las figuras de este artículo se grafican con dicha base de cálculo. 
significativas y reportan los signos predichos en la teoría. Los coeficientes positivos de las variables hombre y formal permiten cuantificar la brecha de género y el premio por formalidad en toda la distribución.

En cuanto a los premios a las calificaciones ocupacionales, se verifican las desigualdades intragrupo, expresadas en términos intercuantílicos (a lo largo de las distribuciones salariales) solo en las categorías técnica y profesional los mismos se asocian a una mayor dispersión salarial, con diferencias estadísticamente significativas entre cuantiles del salario ${ }^{8}$. En ambos niveles. mayor el salario del trabajador, mayor la prima, lo que se verifica en todo el período y en casi todos los intervalos especificados en la tabla. Por el contrario, en las calificaciones operativas, solo en 2004 y 2009 se registran diferencias en algunos tramos (el rango intercuartílico) de la serie de salarios.

Desde otra dimensión, se reportan brechas en las primas entre niveles de calificación (asociada a desigualdad entre grupos), las que resultaron estadísticamente significativas en los test realizados a distintos puntos de la distribución ${ }^{9}$. Se trata de la brecha por calificación operativa, que expresa el premio entre quienes poseen dichas habilidades o conocimientos y quienes no poseen calificación alguna; un premio más alto relacionado a calificaciones técnicas; la brecha entre premios a la calificación técnica y la operativa, y finalmente la brecha profesional-técnica. Estos resultados se encuentran en línea con Casal et al. (2016) y Groisman y Marshall (2015) y tienen su razonamiento en el tiempo que debe destinar el trabajador para adquirir las respectivas calificaciones, por lo que los premios diferenciales implican un reconocimiento a estas habilidades y conocimientos adquiridos.

En términos temporales, los test realizados confirman que las desigualdades entre- e intracalificaciones se moderan hacia 2014. La menor dispersión salarial en el período conlleva a una caída tanto en las brechas de las primas entre niveles de calificación como en las brechas intercuantílicas en cada uno de los niveles de calificación. En el primer caso, hacia 2014 se verifican dos tendencias contrapuestas: se elevan las primas a las calificaciones operativas y se reducen las primas a las calificaciones técnicas y profesionales, modificaciones que se dan en todos los cuantiles bajo estimación. En el segundo caso, la desigualdad intragrupo

8 Por razones de espacio, no se incluyen: los test de diferencias entre coeficientes, ni la tabla de regresión de premios salariales por género, si bien se encuentran disponibles a pedido.

9 Se testearon las diferencias entre los premios por nivel de calificación estimados sobre los percentiles 10,50 y 90 . 


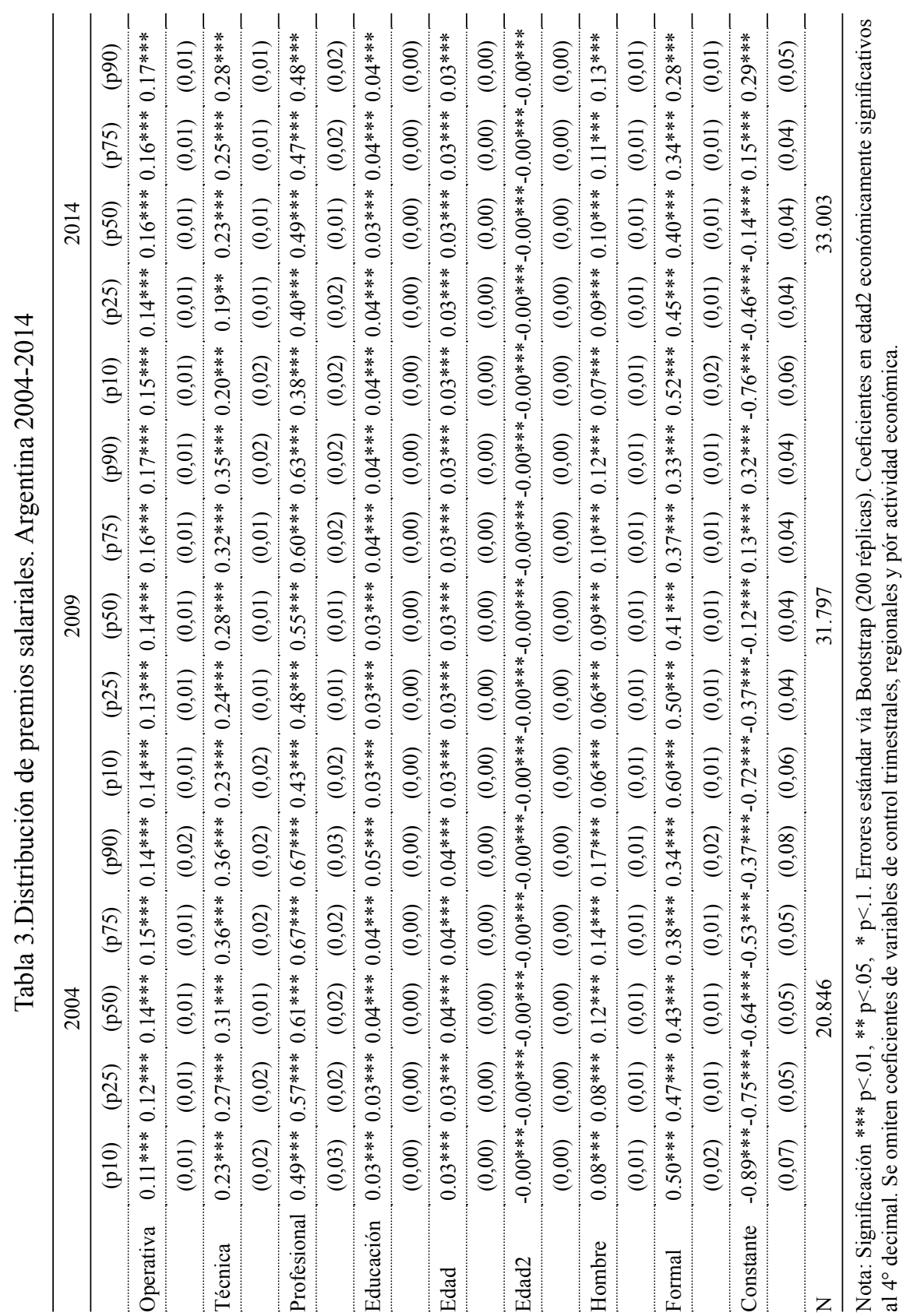


se ve aminorada también. En las calificaciones operativas, a comienzos del período, se registran brechas en las primas de manera parcial (rango p25-p75). En 2014 se anula toda diferencia, resultando en premios uniformes a lo largo de la distribución salarial. En el caso de las calificaciones técnicas y profesionales, si bien las brechas intercuantílicas se contraen significativamente, no alcanzan a eliminarse.

En cuanto a los retornos a la educación, entre 2004 y 2014, la desigualdad intercuantílica se reduce, logrando uniformar los diferenciales salariales hacia fines del período.

La caída en la brecha entre los premios por calificación junto a una menor desigualdad intercuantílica es reportada también en Alejo y Casanova (2016). Al combinar estos hechos con los cambios respecto a la educación de los trabajadores - mejora en los niveles educativos y caída en los retornos-, se identifica una mayor participación de las calificaciones ocupacionales que la educación en la reducción de la desigualdad en el período, en acuerdo con Groisman (2014). Sobre esto, se ensayan dos interpretaciones según el tramo de la distribución salarial. Primero, en el caso de los trabajadores con mayores salarios, la sobreeducación (Groisman \& Marshall, 2015) y sobrecalificación de la fuerza laboral (Jiménez, $2015)$, producto de una oferta de trabajo con altos niveles educativos y de calificación que no puede ser captada por el crecimiento en la demanda. Segundo, respecto a los trabajadores cuyos salarios se ubican en los tramos inferiores de la distribución, el importante proceso de formalización laboral y la mayor incidencia de instituciones como el salario mínimo y las negociaciones colectivas sobre la determinación de los salarios (Amarante \& Arim, 2015; Beccaria, Maurizio, \& Vázquez, 2015; Marshall, 2019).

\section{III.3. Premios a las calificaciones ocupacionales por género}

Finalmente, en esta sección se muestran los resultados del modelo salarial de regresión por cuantiles que interacciona la educación y calificaciones ocupacionales con la condición de género. El propósito de este ejercicio es evaluar cómo las diferencias en las condiciones laborales de hombres y mujeres inciden en la distribución de premios salariales a las calificaciones, con énfasis en su dimensión ocupacional. Los mismos se muestran en la figura 3.

Los resultados de este modelo permiten verificar que los premios a las calificaciones ocupacionales en el grupo femenino resultan menores que los percibidos en el masculino, resultado que se da en todos los niveles. Además, se 
confirma la hipótesis de que, a mayor nivel de calificación, mayor es el premio salarial, si bien en el grupo femenino las brechas entre premios por calificación resultan de menor cuantía. En términos de la desigualdad al interior de los grupos o desigualdad intercuantílica, en el período se observa una caída en la dispersión salarial, común a los tres tipos de calificación y entre géneros.

En primer lugar, los premios a las calificaciones profesionales, técnicas y operativas que perciben las mujeres resultan menores a los que perciben los hombres. Estas diferencias resultan significativas en todos los niveles de calificación. Si bien no se identifican antecedentes empíricos de primas por calificación según género en Argentina, estudios basados en descomposición de brecha de género muestran las diferencias atribuibles a calificaciones que confirman el pago diferencialmente menor en mujeres que en hombres (Esquivel, 2007; Fernandez, 2009; Kř́žková, Penner, \& Petersen, 2010; Bacolod, 2017).

En segundo lugar, al igual que en el modelo sin interacciones, las tareas profesionales retribuyen tanto a hombres como a mujeres de mayores salarios que las tareas técnicas, y estas últimas lo hacen más que las operativas. Estas brechas interocupacionales resultan estadísticamente diferentes a cero, con una significación del $1 \%$ en ambos géneros ${ }^{10}$. $\mathrm{Al}$ combinar este resultado con la brecha entre géneros, se puede deducir por qué, entre mujeres, las brechas entre premios por calificación resultan menores que entre sus pares masculinos. Esta convergencia vertical entre las primas por calificación en mujeres lleva a que calificaciones técnicas y operativas se crucen a los cuantiles más bajos de la distribución salarial.

A nivel intragrupo (al interior de cada género y de cada nivel de calificación), en ambos géneros las distribuciones de premios a las calificaciones ocupacionales tienden a una menor desigualdad entre cuantiles a medida que se avanza en el período. Las series de calificaciones operativas se muestran como aquellas que más uniformidad aportan a la estructura salarial, especialmente en el grupo femenino, lo cual se verifica en los test intercuantílicos ${ }^{11}$. Por el contrario, en los niveles de calificación técnica y profesional, la desigualdad intercuantílica se modera parcialmente entre los hombres (sobre el tramo p25-p75), mientras el trabajo femenino sigue registrando diferencias significativas en los tramos más amplios de la distribución (en el tramo p10-p90).

10 La única excepción se da entre los premios a las calificaciones técnicas y operativas, en el cuantil más bajo de la distribución salarial de mujeres (p10).

11 En 2004 se identifica desigualdad intercuantílica en el tramo intercuartílico, y en 2014 se registran diferencias en la segunda mitad de la distribución. 
Figura 3. Premios a las calificaciones ocupacionales según género. Argentina

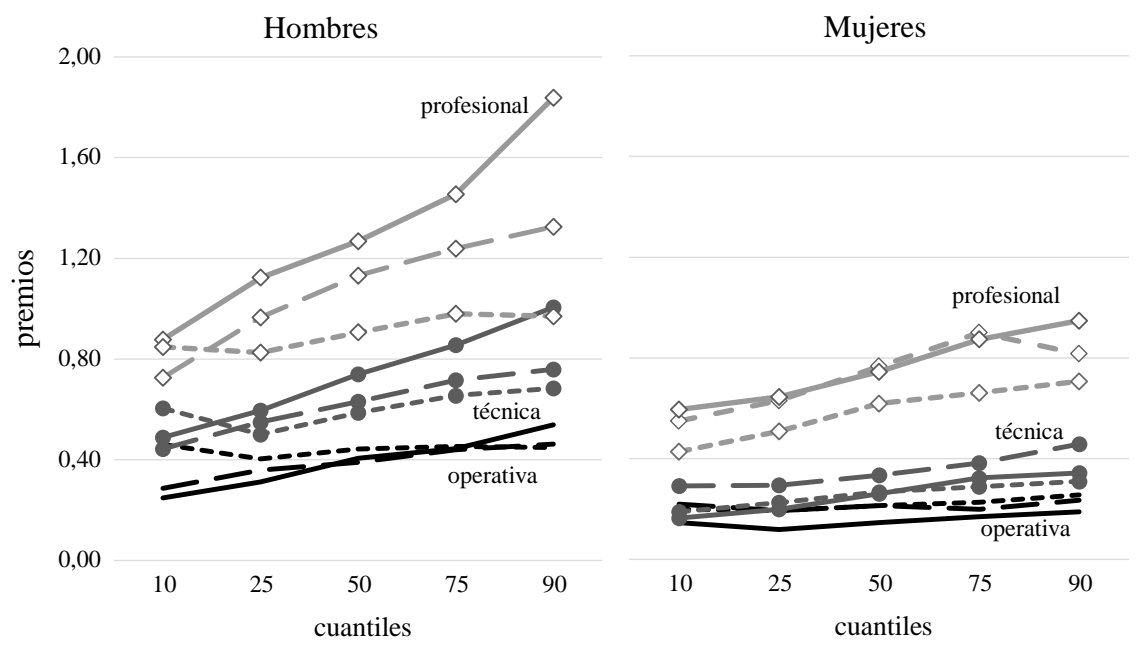

Fuente: elaboración propia con datos de la EPHC (INDEC).

\section{CONCLUSIONES}

Los resultados de este artículo permiten concluir que una apropiada explicación de la desigualdad salarial en Argentina desde la perspectiva de las calificaciones requiere no solo reconocer sino también explicitar que, en buena medida, al trabajador se le paga por las tareas que realiza, de acuerdo a la ocupación que ejerce con las calificaciones que ha adquirido para ocupar este puesto de trabajo. Más aún, que por calificaciones debería considerarse no solo la dimensión educativa, sino en especial las habilidades y conocimientos específicos para ejercer dicha ocupación.

En este artículo se examinó la desigualdad salarial asociada a las calificaciones ocupacionales de los trabajadores argentinos durante la etapa posterior al fin del Plan de Convertibilidad. Los resultados permiten verificar la hipótesis de que los premios a las calificaciones ocupacionales se vinculan con una mayor desigualdad salarial. Más aún, este vínculo se puede verificar considerando distintas dimensiones de la desigualdad salarial: entre grupos o niveles de calificación, e intragrupos o hacia dentro de las calificaciones. En el primer caso, analizando las 
brechas por calificación se verifica que, a mayor calificación, mayor es el salario, lo que evidencia la vigencia de una estructura de premios salariales asociada a ciertos tipos de ocupación. Este resultado es independiente del modelo estimado y de la posición en la distribución salarial. En el segundo caso, referido a los tramos de la distribución salarial en cada nivel de calificación, se registra también una asociación directa entre los premios y la desigualdad, en este caso intercuantílica.

Por otro lado, considerando los premios según el género, los mismos resultan significativamente mayores para el género masculino, condición que se repite en todos los tramos salariales, constituyéndose las calificaciones ocupacionales como factores que pueden acentuar las brechas salariales por género.

En cuanto a la evolución en el período, se confirma la hipótesis de una reducción en los premios a las calificaciones y los retornos a la educación hacia fines del período. Entre las razones que permiten interpretar estos cambios se encuentran las instituciones laborales (salario mínimo y negociaciones colectivas) y los cambios en la composición del mercado de trabajo por políticas de recuperación y registración del empleo. Ambos podrían explicar las mejoras relativas de los asalariados con menores salarios o menores calificaciones. En el otro extremo, la caída en los premios salariales asociados a mayores niveles de calificación y, complementariamente, a los salarios más altos de cada distribución podría estar asociada a un desbalance educativo o de calificaciones que conlleva penalidades en términos relativos.

Cabe mencionar ciertas limitaciones en el análisis. Primero, el análisis de los vínculos entre calificaciones y desigualdad salarial no puede interpretarse en términos de relaciones causales. Las múltiples conexiones entre variables consideradas impiden plantear, para la estrategia empírica adoptada, una relación de causalidad en un solo sentido, ya que ignoraría los potenciales fenómenos de causalidad inversa identificadas en las ecuaciones de Mincer. Dado que la técnica de regresión por cuantiles con variables instrumentales es incipiente en econometría y excede los objetivos aquí planteados, se deja en claro la posible existencia de un sesgo que sobrestime los efectos positivos en los premios salariales. Segundo, resulta necesario avanzar con el tratamiento de potenciales sesgos de selección. Esto implicaría adaptar el planteo de los modelos de selección al contexto de regresiones por cuantiles, lo cual requiere el empleo de algoritmos apropiados para la/s regla/s de selección, tema a considerar a futuro en esta línea de investigación. Tercero, la incorporación de las calificaciones ocupacionales responde a una configuración en buena parte simplificada de cómo se valoran las mismas en el mercado laboral. 
Un desarrollo más elaborado en este aspecto sería saludable, si bien los resultados expuestos aquí cobran relevancia al considerar otras dimensiones no asociadas estrictamente a lo educativo en los mecanismos de determinación salarial. Cuarto, por razones de espacio no se consideraron los condicionamientos que le imprime la segmentación de mercados laborales a los premios salariales. Los premios por formalidad y su estructura serán incluidos en una futura investigación.

A partir de este artículo, se pueden visualizar ciertas implicancias a nivel de política pública. Primero, si el salario no solo depende de la educación, y se reconoce al mismo como un pago por las tareas realizadas en una ocupación determinada, se abre un abanico de políticas que pueden mejorar el acceso de los trabajadores según sus calificaciones a los empleos que las requieren (buscando mitigar posibles desbalances en las calificaciones y favorecer el flujo de información entre trabajadores y firmas atendiendo a necesidades en un mediano plazo). Segundo, la evidencia de la segregación ocupacional por género derivada de la brecha identificada en las primas por calificación permite visualizar que buena parte de las políticas de igualdad de género deberían enfocarse en el aspecto laboral, con acciones positivas de intervención estatal que eviten la discriminación en el acceso a ciertas ocupaciones o ciertas jerarquías. Tercero, la introducción de esta temática permite enfocar el estudio de los aspectos distributivos y laborales hacia el mundo de las ocupaciones y las tareas de los trabajadores, cuestiones críticas en la elaboración de una estrategia de desarrollo productivo y tecnológico que contemple una configuración factible del futuro del trabajo en Argentina

\section{REFERENCIAS BIBLIOGRÁFICAS}

Acemoglu, D. (2002). Technical change, inequality, and labor market. Journal of economic literature, 40 (1), 7-72.

Acemoglu, D., \& Autor, D. (2011). Skills, tasks and technologies: Implications for employment and earnings. En Card, D., \& Ashenfelter, O. (eds.). Handbook of labor economics (Vol. 4, Part B, pp. 1043-1171).

Alejo, J. (2018). Educación y Desigualdad Salarial: una descomposición basada en dos interpretaciones de la ecuación de Mincer. Evidencia para Argentina. En Escardíbul, J.-O., \& Choi Á. (eds.). Investigaciones de Economía de la Educación (Vol. 13, pp. 293-314). Barcelona: Asociación de Economía de la Educación. 
Alejo, J., \& Casanova, L. (2016). Negociación colectiva y cambios distributivos en los ingresos laborales en argentina. Revista de Economía Política de Buenos Aires 15 (10), 65-97.

Aliaga, R., \& Montoya, S. (1998). Tasas de retorno a la inversión en capital humano. Argentina 1990-1998. Revista Estudios, 21 (86), 95-117.

Alzúa, M. L. (2008). Are informal workers secondary workers?: Evidence for Argentina. (Documento de trabajo No 73). La Plata: CEDLAS.

Alzúa, M. L., Gasparini, L., \& Haimovich, F. (2015). Education Reform and Labor Market Outcomes: The Case of Argentina's Ley Federal De Educación. Journal of Applied Economics, 18 (1), 21-43.

Amarante, V., \& Arim, R. (2015). Desigualdad de las remuneraciones e informalidad: breve revisión de literatura y marco analítico. En Amarante, V., \& Arim, R. (eds.). Desigualdad e informalidad. Un análisis de cinco experiencias latinoamericanas (Vol. 133, pp. 19-35). Santiago de Chile: CEPAL.

Autor, D., \& Handel, M. (2013). Putting tasks to the test: Human capital, job tasks, and wages. Journal of Labor Economics, 31 (S1), S59-S96.

Autor, D., Levy, F., \& Murnane, R. (2003). The skill content of recent technological change: An empirical exploration. The Quarterly Journal of Economics, 118 (4), 1279-1333.

Avent-Holt, D., Henriksen, L. F., Hägglund, A. E., Jung, J., Kodama, N., Melzer, S. M., ... Tomaskovic-Devey, D. (2019). Occupations, workplaces or jobs?: An exploration of stratification contexts using administrative data. Research in Social Stratification and Mobility, [100456]. https://doi.org/10.1016/j. rssm.2019.100456

Bacolod, M. (2017). Skills, the gender wage gap and cities. Journal of Regional Science, 57 (2), 290-318.

Beccaria, L., \& Groisman, F. (2015). Informalidad y segmentación del mercado laboral: el caso de la Argentina. Revista CEPAL (№ 117), 127-143.

Beccaria, L., Maurizio, R., \& Vázquez, G. (2015). Recent decline in wage inequality and formalization of the labour market in Argentina. International Review of Applied Economics, 29(5), 677-700.

Becker, G. S. (1962). Investment in Human Capital: A Theoretical Analysis. Journal of Political Economy, 70 (5), 9-49.

Becker, G. S. (1964). Human capital. A Theoretical and Empirical Analysis with Special References to Education. London: The University of Chicago Press. The National Bureau of Economic Research.

Becker, G. S., \& Chiswick, B. R. (1966). Education and the Distribution of Earnings. The American Economic Review, 56 (1/2), 358-369. 
Berniell, I., Berniell, L., de la Mata, D., Edo, M., \& Marchionni, M. (2018). Motherhood and the missing women in the labor market. CAF - Development Bank of Latin America (No. 2018/13).

Card, D., \& DiNardo, J. E. (2002). Skill-biased technological change and rising wage inequality: Some problems and puzzles. Journal of Labor Economics, 20 (4), 733-783.

Card, D., \& Lemieux, T. (2001). Can falling supply explain the rising return to college for younger men? A cohort-based analysis. The Quarterly Journal of Economics, 116 (2), 705-746.

Casal, M., Paz Terán, C., \& Paz, J. (2016). Educación y desigualdad. Evolución en Argentina en los últimos 20 años (1995-2015). Paper presented at the LI Reunión Anual AAEP, Tucumán.

Ciaschi, M., Galeano, L., \& Gasparini, L. (2019). Estructura productiva y desigualdad salarial: evidencia para América Latina. (Documento de Trabajo No 250). La Plata: CEDLAS.

De Beyer, J., \& Knight, J. B. (1989). The role of occupation in the determination of wages. Oxford economic papers, 41 (3), 595-618.

De Santis, M., Gáname, M. C., \& Moncarz, P. E. (2018). The impact of overeducation on wages of recent economic sciences graduates. Trabajo presentado en LIII Reunión de la AAEP, La Plata.

Esquivel, V. (2007). Género y Diferenciales de Salarios en la Argentina. En Novick, M. \& Palomino, H. (coords.). Estructura Productiva y Empleo: Un Enfoque Transversal (pp. 363-392). Buenos Aires: Ministerio de Trabajo, Empleo y Seguridad Social.

Fernandez, J. L. (2009). Intra-occupational gender earnings gaps in Malaysia. Jurnal Kemanusiaan, 7 (2).

Galiani, S., Cruces, G., Acosta, P., \& Gasparini, L. C. (2017). Educational upgrading and returns to skills in Latin America: Evidence from a supplydemand framework. (Working Paper Series No. w24015). Massachusetts: National Bureau of Economic Research.

Gasparini, L., \& Cruces, G. (2009). A Distribution in Motion: The Case of Argentina: A Review of the Empirical Evidence. Research for Public Policy, Inclusive Development, (ID 06-2009). New York: BLAC-UNDP.

Goldin, C., \& Katz, L. F. (2009). The race between education and technology. Cambridge, Massachusetts: Belknap Press for Harvard University Press.

Gómez, M. C. (2020). Elementos de poder sindical y sus efectos en la desigualdad salarial argentina. Afiliación, negociación colectiva y conflictos laborales. Revista CIFE: Lecturas de Economía Social, 22 (36). 
Groisman, F. (2014). Empleo, salarios y desigualdad en Argentina: análisis de los determinantes distributivos. Problemas del desarrollo, 45 (177), 59-86.

Groisman, F., \& Marshall, A. (2015). Educación, demanda de calificaciones y salarios relativos: el caso argentino, 2004-2011. Desarrollo Económico, 55 (216), 227-243.

Groisman, F. A. (2015). Informalidad, ingresos laborales y papel del salario mínimo En Bertranou, F. \& Casanova, L. (coords.). Caminos hacia la formalización laboral en Argentina (Cap. 4, pp. 103-117). Buenos Aires, Argentina: Organización Internacional del Trabajo.

Hartog, J. (2000). Over-education and earnings: where are we, where should we go? Economics of education review, 19 (2), 131-147.

Heckman, J. J., \& Honore, B. E. (1990). The empirical content of the Roy model. Econometrica: Journal of the Econometric Society, 58 (5), 1121-1149.

INDEC (2002). Paridades de poder de compra del consumidor (PPCC). Dirección de Índices de Precios de Consumo. Instituto Nacional de Estadística y Censos.

INDEC (2018). Clasificador Nacional de Ocupaciones. Dirección Nacional de Metodología Estadística. Instituto Nacional de Estadística y Censos.

Jiménez, M. (2015). Desbalance de calificaciones, polarización en la creación de empleo e informalidad: evidencia para Argentina. En Bertranou, F. \& Casanova, L. (coords.), Caminos hacia la formalización laboral en Argentina (Cap. 7, pp. 157-181). Buenos Aires: Organización Internacional del Trabajo.

Joll, C., McKenna, C., McNabb, R., \& Shorey, J. (1983). Developments in labour market analysis. Londres: George Allen \& Unwith.

Katz, L. F., \& Murphy, K. M. (1992). Changes in Relative Wages, 1963-1987: Supply and Demand Factors. The Quarterly Journal of Economics, 107 (1), 35-78.

Koenker, R., \& Bassett, G. (1978). Regression quantiles. Econometrica: Journal of Econometric Society, 33-50.

Kř́žzová, A., Penner, A. M., \& Petersen, T. (2010). The legacy of equality and the weakness of law: Within-job gender wage inequality in the Czech Republic. European Sociological Review, 26 (1), 83-95.

Kunst, D., Freeman, R. B., \& Oostendorp, R. (2020). Occupational Skill Premia around the World. (Working Paper Series, No. 26863). Massachusetts: National Bureau of Economic Research.

Machado, J. A., \& Mata, J. (2005). Counterfactual decomposition of changes in wage distributions using quantile regression. Journal of applied Econometrics, 20 (4), 445-465. 
Marchionni, M., Gasparini, L., \& Edo, M. (2019). Brechas de género en América Latina. Un estado de situación Caracas: CAF. Recuperado de http://scioteca. caf.com/handle/123456789/1401

Marshall, A. (2019). Salario mínimo y negociación colectiva e la determinación salarial: interacciones entre políticas salariales y posturas sindicales en la Argentina y Uruguay. Trabajo y sociedad: Indagaciones sobre el empleo, la cultura y las prácticas políticas en sociedades segmentadas (32), 79-101.

Mincer, J. (1974). Schooling, Experience, and Earnings. Human Behavior \& Social Institutions. New York: National Bureau of Economic Research.

Pal, J. M. (2019). Brechas de género: una exploración más allá de la media. (Documento de Trabajo No 255). La Plata: CEDLAS.

Paz, J. A. (2013). Segmentación del mercado de trabajo en la Argentina. Revista Desarrollo y Sociedad, 72, 105-156.

Paz, J. A. (2015). Informalidad laboral y segmentación en la Argentina. Dimensión regional. En Bertranou, F., \& Casanova, L. (eds.). Caminos hacia la formalización laboral en Argentina. Buenos Aires: OIT.

Polachek, S. W. (1981). Occupational self-selection: A human capital approach to sex differences in occupational structure. The Review of Economics and statistics, 60-69.

Quinn, M. A., \& Rubb, S. (2006). Mexico's labor market: The importance of education-occupation matching on wages and productivity in developing countries. Economics of education review, 25 (2), 147-156.

Roy, A. D. (1951). Some thoughts on the distribution of earnings. Oxford economic papers, 3 (2), 135-146.

Schultz, T. W. (1961). Investment in human capital. The American Economic Review, 51 (1), 1-17.

Villanueva, A., \& Lin, K.-H. (2019). Motherhood Wage Penalties in Latin America: The Significance of Labor Informality. Social Forces. Recuperado de https:// doi.org/10.1093/sf/soz142

(C) 2020 por los autores; licencia no exclusiva otorgada a la revista Estudios económicos. Este artículo es de acceso abierto y distribuido bajo los términos y condiciones de una licencia Atribución-No Comercial 4.0 Internacional (CC BY-NC 4.0) de Creative Commons. Para ver una copia de esta licencia, visite http://creativecommons.org/licenses/by-nc/4.0 
